\title{
A systematic localized approximation scheme for the coupled-cluster treatment of quantum spin systems
}

\author{
R F Bishop, J B Parkinson and Yang Xian \\ Department of Mathematics, UMIST, PO Box 88, Manchester M60 1QD, UK
}

Received 26 February 1992

\begin{abstract}
Within the framework of the coupled-cluster method a systematic localized approximation scheme, the so-called LSUBn scheme, is developed and applied to anisotropic quantum spin chains. We use computer generation of the terms in the coupled non-linear equations to obtain successive approximations up to onder $n=8$. The results for the ground-state energy show systematic and rapid convergence in the Ising-like region. The method also gives good results in the planar region where perturbation theory is not valid, and only breaks down for values of the anisotropy parameter $\Delta \lesssim-0.5$. We show that for a given value of $n$ the LSUB $n$ scheme reproduces exactly the corresponding $n$th order of large- $\Delta$ perturbation theory around the Ising limit.
\end{abstract}

\section{Introduction}

Although the coupled-cluster method (CCM) is firmly established as one of the most powerful methods of quantum many-body theory (see Bishop and Kümmel (1987) and Bishop (1991) for introductory reviews), it is only recently that it has been applied to quantum spin systems. This was first done by Roger and Hetherington (1990) and then developed in earlier papers by the present authors (Bishop et al 1991a, b, henceforth referred to as I and II respectively). In these papers the potential of the $C C M$ as applied to antiferromagnetic quantum spin systems was demonstrated. We introduced a variety of approximation schemes and showed that even in rather low orders these were capable of obtaining very encouraging results when compared with known exact results for $s=\frac{1}{2}$ quantum spin chains. The Hamiltonian considered was the $X X Z$ or anisotropic Heisenberg model:

$$
\mathcal{H}=\frac{1}{2} \sum_{l} \sum_{\rho}\left[\Delta s_{l}^{z} s_{l+\rho}^{z}+s_{l}^{x} s_{l+\rho}^{x}+s_{l}^{y} s_{l+\rho}^{y}\right]
$$

where the sum over $l$ is over all $N$ atoms with periodic boundary conditions, and the sum over $\rho$ is over nearest neighbours. This model in one dimension is integrable using the Bethe ansatz (Bethe 1931, Orbach 1958, Yang and Yang 1966a, b). We calculated the ground-state energy per spin, the sublattice magnetization, the spinspin correlation function and the excited-state energies as functions of $\Delta$.

Of course, the CCM is applicable to non-integrable systems just as easily as to integrable ones and in the above references we also investigated $2 \mathrm{D}$ systems described by (1). Furthermore, we have recently obtained results for the similar 1D system with 
$s \geqslant 1$ (Bishop et al 1992), which is also non-integrable. However, in order for our results to be useful in practice we need to have some measure of the limitations of any particular approximation scheme, and to confirm that successive terms in such a scheme do indeed converge over some range of the parameters. For this reason we present results in this paper in which one particular scheme, the LSUBn scheme, is taken to higher order. Again, for comparison with exact results, we consider the ID $s=\frac{1}{2}$ Bethe ansatz integrable Hamiltonian of (1). We shall concentrate on the ground-state energy per spin for this system.

\section{The $L S U B n$ approximation}

The starting point of the $\mathrm{OCM}$ is the choice of an uncorrelated model or reference state $|\Phi\rangle$ which we shall take as the usual two-sublattice Néel state. Following Roger and Hetherington, for ease of later description we perform a notional rotation of $180^{\circ}$ on one sublattice so that the model state may be referred to as having all spins pointing down.

We shall work with Pauli spin operators $\sigma_{i}^{\alpha}$, related to the spin angular momentum operators in the usual way: $\sigma_{i}^{\alpha}=2 s_{i}^{\alpha}, \alpha=x, y, z$. Defining raising and lowering operators $\sigma_{i}^{ \pm}=\frac{1}{2}\left(\sigma_{i}^{x} \pm \mathrm{i} \sigma_{i}^{y}\right)$ for index $i$ on the 'down' sublattice and $\sigma_{j}^{ \pm}=\frac{1}{2}\left(-\sigma_{j}^{x} \pm\right.$ $\left.\mathrm{i} \sigma_{j}^{y}\right)$ for index $j$ on the 'up' sublattice, then $\sigma_{l}^{-}|\Phi\rangle=0$ for index $l$ on either sublattice, while $\sigma_{l}^{+}|\Phi\rangle$ is a state with the $l$ th spin reversed with respect to the model state (and see II for further details). The Hamiltonian of (1) becomes

$$
\mathcal{H}=-\sum_{l}\left[(\Delta / 4) \sigma_{l}^{z} \sigma_{l+1}^{z}+\frac{1}{2}\left(\sigma_{l}^{+} \sigma_{l+1}^{+}+\sigma_{l}^{-} \sigma_{l+1}^{-}\right)\right] .
$$

In the $\mathrm{CCM}$ the true ground state is written as

$$
|\Psi\rangle=\mathrm{e}^{s}|\Phi\rangle
$$

The $\propto \mathrm{CM}$ correlation operator $S$ is constructed entirely out of creation operators with respect to the model state, i.e. out of a sum of terms containing all possible combinations of the $\left\{\sigma_{i}^{+}\right\}$creation operators consistent with the conserved quantities. For the ground state of (1) we require that

$$
s_{\mathrm{T}}^{z} \equiv \sum_{l} s_{l}^{z}=0
$$

Any particular approximation consists of selecting a subset of these terms.

The particular choice known as the LSUB $n$ scheme, first described in I, includes all possible terms that involve creation operators acting wholly within a 'locale' of $n$ adjacent sites. In each term there must be an equal number of spin fips with respect to the model state on each sublattice since $s_{\mathrm{T}}^{z}$ is a conserved quantum number, as noted above. We therefore write for $S$ within this scheme

$$
S=\sum_{k=1}^{n / 2} S_{2 k}
$$


where

$$
S_{2 k}=(k !)^{-2} \sum_{\text {all subscripts }} S_{i_{1} i_{2} \ldots i_{k} j_{2} j_{2} \ldots j_{k}} \sigma_{i_{1}}^{+} \sigma_{i_{2}}^{+} \ldots \sigma_{i_{k}}^{+} \sigma_{j_{1}}^{+} \sigma_{j_{2}}^{+} \ldots \sigma_{j_{k}}^{+}
$$

In this expression the indices $\left\{i_{n}\right\}$ run over all sites on one of the two sublattices, while the indices $\left\{j_{n}\right\}$ refer to the opposite sublattice. The cluster configuration coefficients

$$
\left\{S_{i_{1} i_{2} \ldots i_{k} ; j_{1} j_{2} \ldots j_{k}}\right\}
$$

are defined to be invariant with respect to permutations of the indices $\left\{i_{n} ; n=\right.$ $1,2, \ldots, k\}$ amongst themselves and of the indices $\left\{j_{n} ; n=1,2, \ldots, k\right\}$ amongst themselves. The configuration coefficients also share the various symmetries of the lattice, ie., in ID, both translational and reflection symmetry. Thus, for example, for the compact four-spin cluster, we have

$$
S_{i, i+2 ; i+1, i+3}=S_{i+2, i ; i+1, i+3}=S_{i, i+2 ; i-1, i+1} \quad \text { etc. }
$$

Because of the translational symmetry the coefficients in the sums are independent of $i_{1}$. Choosing $i_{1}=0$ we introduce a simplified notation:

$$
f_{0 \alpha \beta \gamma \delta \ldots \omega} \boxminus S_{i_{1} i_{2} \ldots i_{k} ; j_{1} j_{2} \ldots j_{k}}
$$

where the $2 k-1$ indices $\alpha<\beta<\gamma<\delta<\ldots<\omega$ are the ordered set of differences $\left(i_{2}-i_{1}\right),\left(i_{3}-i_{1}\right), \ldots,\left(i_{k}-i_{1}\right),\left(j_{1}-i_{1}\right), \ldots,\left(j_{k}-i_{1}\right)$. For consistency with the notation of previous papers we also use $b_{n} \equiv f_{0 n}$, and $g_{n} \equiv f_{01234 \ldots n-1}$ for the configuration in which all the spins in a block of $n$ adjacent sites are flipped. With this notation the various independent terms included in the LSUB $n$ approximation for $2 \leqslant n \leqslant 8$ are as follows:

LSUB2: $b_{1}$

LSUB3: $b_{1}$

LSUBA: $b_{1}, b_{3}, g_{4}$

LSUBS: $b_{1}, b_{3}, g_{4}, f_{0134}$

LSUB6: LSUB5 plus $b_{5}, f_{0125}, f_{0235}, f_{0145}, g_{6}$

LSUB7: LSUB6 plus $f_{0136}, f_{0156}, f_{012356}$

LSUB8: LSUB7 plus $b_{7}, f_{0127}, f_{0147}, f_{0167}, f_{0237}, f_{0257}, f_{0347}, f_{012347}, f_{012367}, f_{012457}$, $f_{012567}, f_{013467}, f_{023457}, g_{8}$.

These terms are displayed diagrammatically in figure 1 . All coefficients that are not explicitly included at any given level of approximation are taken to be zero, except that use has been made of the fact that terms that are identical under reflection symmetry are equal, and hence only one term of each such pair needs to be considered explicitly. Thus, for example, $f_{0345} \equiv f_{0125}$ is also retained in the LSUBn approximation with $n>5$.

Note that there is no LSUB1 approximation as there must always be an even number of creation operators in each term of $S$, and for similar reasons the LSUB3 approximation is identical to LSUB2. Also, for small values of $n$, more terms are added as $n$ is increased by unity from odd values of $n$ than from even values of $n$. This combinatorial effect is due to the fact that for odd values of $n$ the locale does not include equal numbers of sites on each sublattice. 


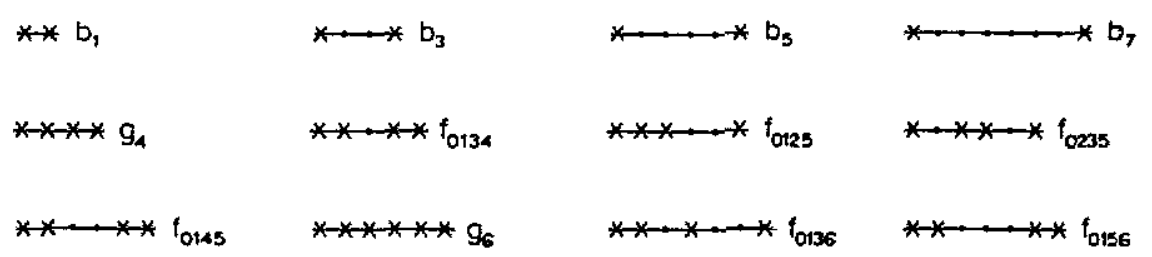

$* x-*-x \rightarrow * * * f_{012356}$
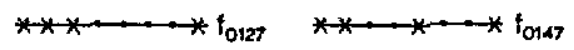

$* *-\ldots+\ldots+\ldots+0167$

$* * \ldots \ldots+\ldots$ to237
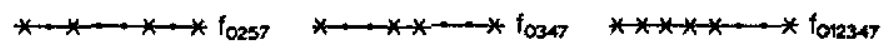

$x-x-x-x-\ldots-x-x+0+2367$

$* x-x \rightarrow-x-* * 0_{012457}$

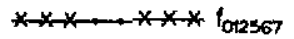

$* *-x-x-x-* * f_{013467}$

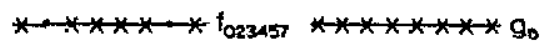

Figure 1. The 26 independent configurations required for the LSUBs approximation. Each cross $(x)$ indicates a site that is flipped relative to the Néel state; while a dot $(\cdot)$ indicates an unflipped site. Al sites not explicitly shown are unflipped. Each configuration may appear at any position atong the chain. Note that for the asymmetric onfigurations we also retain their (identical) reflection-symmetry counterparts.

By taking the translational and reflection symmetry into account explicitly, it is possible to show that the number $N_{n}$ of independent configuration coefficients that need to be retained at the LSUB $n$ level of approximation is given by

$$
N_{n}=\frac{1}{2}\left\{\left(\begin{array}{c}
n-1 \\
{[n / 2]-1}
\end{array}\right)+2^{[n / 2]}-2+\left(\begin{array}{c}
{[(n-1) / 2]} \\
{[n / 4]}
\end{array}\right)\right\}
$$

where $[p]$ denotes the integer part of the number $p$, and $\left(\begin{array}{c}m \\ n\end{array}\right)$ is the usual combinatorial factor,

$$
\left(\begin{array}{c}
m \\
n
\end{array}\right)=\frac{m !}{n !(m-n) !}
$$

It is clear from the form of the Hamiltonian that an lSUBn sequence in which only even values of $n$ are considered is in some ways more natural than the more general sequence which includes all values of $n$. The numerical results presented in section 3 reflect this.

Starting with the Schrödinger equation

$$
\mathcal{H}|\Psi\rangle=E_{\mathrm{g}}|\Psi\rangle
$$

the ground-state energy, $E_{g}$, is obtained by operating on the left with $\langle\Phi|$. For all approximation schemes this yields

$$
E_{\mathrm{g}} / N=-\frac{1}{4}\left(\Delta+2 b_{1}\right)
$$

reflecting the nearest-neighbour interaction form of $\mathcal{H}$. Indeed, equation (8) is ${ }_{\mathrm{f}}$ an exact equation in terms of the compact two-spin-fip configuration coefficient $b_{1}$. 
Furthermore, a set of coupled non-linear equations for the cluster configuration coefficients is obtained by operating on the left of the Schrödinger equation with $\langle\Phi| C_{l}$, where $C_{I}$ is the Hermitian conjugate of one of the strings of creation operators (combinations of $\sigma_{i}^{+}$) present in $S$.

The simplest approximation, LSUB2, retains only the single term with coefficient $b_{1}$, and leads to a single non-linear equation

$$
-1+2 \Delta b_{1}+3 b_{1}^{2}=0
$$

and hence

$$
E_{\mathrm{g}} / N=-\left[\Delta+2\left(\Delta^{2}+3\right)^{1 / 2}\right] / 12 \text {. }
$$

At $\Delta=1$, for example, this gives $E_{\mathrm{g}} / N=-5 / 12=-0.4167$, compared with the exact result -0.4432 .

In the LSUB4 approximation the three coupled equations are

$$
\begin{aligned}
& -1+2 \Delta b_{1}-2 g_{4}+3 b_{1}^{2}-2 b_{1} b_{3}-2 b_{3}^{2}=0 \\
& 4 \Delta b_{3}-g_{4}-b_{1}^{2}+4 b_{1} b_{3}=0 \\
& \Delta g_{4}-\Delta\left(b_{1}^{2}+2 b_{1} b_{3}\right)+g_{4}\left(4 b_{1}+b_{3}\right)+2 b_{1} b_{3}^{2}=0 .
\end{aligned}
$$

These equations may be called the $b_{1}, b_{3}$, and $g_{4}$ equations respectively, corresponding to the three choices of $C_{I}$ from which they were obtained. They are readily solved in practice by a simple iterative method which also works satisfactorily for the higher approximations. This particular LSUB4 approximation was also considered for the case $\Delta=1$ in the pioneering paper of Roger and Hetherington (1990).

In the LSUBS approximation the four equations are

$$
-1+2 \Delta b_{1}-2 g_{4}-2 f_{0134}+3 b_{1}^{2}-2 b_{1} b_{3}-2 b_{3}^{2}=0
$$

$4 \Delta b_{3}-g_{4}-b_{1}^{2}+4 b_{1} b_{3}=0$

$\Delta g_{4}-\Delta b_{1}\left(b_{1}+2 b_{3}\right)+g_{4}\left(4 b_{1}+b_{3}\right)+f_{0134}\left(b_{1}-b_{3}\right)+2 b_{1} b_{3}^{2}=0$

$2 \Delta f_{0134}-2 \Delta b_{3}^{2}+g_{4}\left(b_{1}+b_{3}\right)+4 b_{1} f_{0134}+2 b_{1}^{2} b_{3}=0$

while for the LSUB6 approximation the nine equations are given in appendix 1 .

It is clear that both the number of equations in the LSUB $n$ approximation scheme and the number of terms in each equation increase rapidly as $n$ increases. Although the equations for $n \leqslant 4$ were obtained directly, an algebraic computational method is required for larger $n$. This uses exactly the same method as in the direct calculation and details are given in appendix 2 . Using this we obtained and solved the equations for $n \leqslant 8$ using a desktop computer, and we note that it should be quite straightforward to go to at least $n=10$ using more powerful computers. 


\section{Results for the ground-state energy}

In table 1 we give the values of $E_{\mathrm{g}} / N$ for various values of $\Delta$ for the LSUBn approximations with $n \leqslant 8$, together with perturbation theory results and the exact results. We note that the LSUB $n$ results for even $n$ form a sequence that appears to converge monotonically to the exact result, for $\Delta \gtrsim 0$. The odd values give a sequence that also converges for approximately the same range of $\Delta$, but that is not as accurate as the even- $n$ sequence for low values of $n$. We believe that this is a combinatorial effect as discussed in the previous section. We also note that the LSUB $n$ numerical results for the odd values $n=3,5,7$ lie everywhere above the exact result. We have been unable to prove that the odd values give an upper bound in general. Nevertheless, the observed difference between the odd- $n$ and even- $n$ sequences suggests that some such general result may be true.

Table 1. Ground-state energy per spin as a function of anisotropy $\Delta$. PTn is the result of perburbation theory to order $\Delta^{-n+1}$, whereas LSUBn represents the present results.

\begin{tabular}{lllllll}
\hline & \multicolumn{6}{c}{$\Delta$} \\
\cline { 2 - 7 } Method & 0.0 & 0.5 & 1.0 & 1.5 & 2.0 & 5.0 \\
\hline LSUB2 & -0.2887 & -0.3412 & -0.4167 & -0.5069 & -0.6076 & -1.2986 \\
LSUB4 & -0.3193 & -0.3692 & -0.4363 & -0.5195 & -0.6155 & -1.2995 \\
LSUBS & -0.3078 & -0.3638 & -0.4339 & -0.5185 & -0.6151 & -1.2995 \\
LSUB6 & -0.3198 & -0.3730 & -0.4400 & -0.5218 & -0.6167 & -1.2995 \\
LSUB7 & -0.3125 & -0.3694 & -0.4385 & -0.5213 & -0.6165 & -1.2995 \\
LSUB8 & -0.3196 & -0.3741 & -0.4414 & -0.5226 & -0.6170 & -1.2995 \\
PT2 & & -0.6250 & -0.5000 & -0.5417 & -0.6250 & -1.3000 \\
PT4 & & -0.1250 & -0.4375 & -0.5232 & -0.6172 & -1.2995 \\
PT6 & & -0.1250 & -0.4375 & -0.5232 & -0.6172 & -1.2995 \\
PT8 & & -0.6250 & -0.4414 & -0.5234 & -0.6172 & -1.2995 \\
Exact & -0.3183 & -0.3750 & -0.4432 & -0.5234 & -0.6172 & -1.2995 \\
\hline
\end{tabular}

Comparing the LSUB $n$ results with $n$ th-order perturbation theory (i.e. up to terms of order $\Delta^{-n+1}$ ) we see that LSUB $n$ produces good results for $0 \lesssim \Delta<1$ where the perturbation sequence in inverse powers of $\Delta$ obviously fails. However, for $\Delta \gtrsim 1$ the LSUB $n$ results are not quite as good as the corresponding results from $n$ th-order perturbation theory, although they clearly converge to the correct result. Nevertheless this observation is somewhat misleading as we show in the next section that the LSUB $n$ approximation identically reproduces the large- $\Delta$ perturbation theory series out to terms of order $\Delta^{-n+1}$.

Our LSUB $n$ approximation scheme does very much better than perturbation theory for smaller $\Delta$, where the latter starts to diverge, because it includes physically important contributions from all orders in $\Delta^{-1}$. We believe the reason it is marginally worse for $\Delta \gtrsim 1$ is a consequence of the abnormally small values of the coefficients in the perturbation series (Walker 1959). Errors in the coefficients of terms that are higher order than $\Delta^{-n+1}$ have a larger effect than might be expected since they cannot reproduce the almost perfect cancellation that leads to the very small actual values of these coefficients.

In figure 2 we plot the ground-state energy per spin as a function of $\Delta$ for the various LSUB $n$ approximations considered. The sequence breaks up in a nonuniform manner for $\Delta \lesssim 0$, in a way that is rather reminiscent of the break-up of 
the perturbation sequence for $\Delta \lesssim 1$ (see also figure 3). Nevertheless it appears that the method is reliable and accurate for all positive $\Delta$ and consequently should be applicable over this range to non-integrable systems such as $2 \mathrm{D} s=\frac{1}{2}$ models and iD $s \geqslant 1$ models. Indeed it appears to be converging satisfactorily even for negative $\Delta \gtrsim-0.5$.

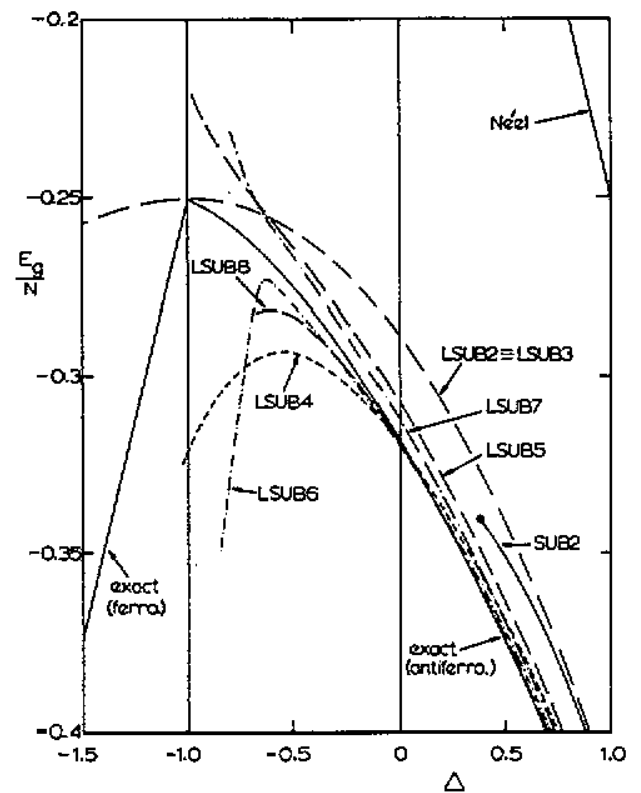

Figure 2. Ground-state energy per spin for the D $X X Z$ model with $s=\frac{1}{2}$ as a function of $\Delta$ showing the exact result, and results for the LSUB $n$ approximation schemes with $n=2,3,4,5,6,7$, 8. Note that LSUB3 is identical to LSUB2. We also show results of the SUB2 scheme described in I and II.

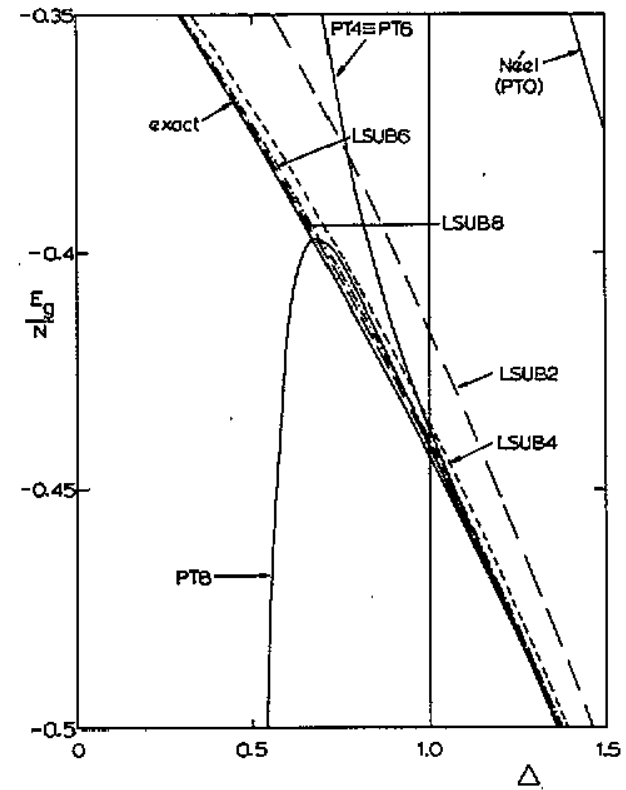

Figure 3. Comparison of the ground-state energy per spin calculated using LSUB $n$ and using PT $n$ for $n=2,4,6,8$. PT $n$ is $n$ th-order perturbation theory, i.e. up to terms of order $\Delta^{-n+1}$. Note that the Néel result is PTO and that the curve for PT2 lies outside the ranges of this figure. Also note that PT4 and PT6 are identical, since the term of order $\Delta^{-5}$ has zero coefficient.

It is interesting to note that exact results on the asymptotic behaviour of the spin-spin correlation function $g_{n} \equiv\left\langle\sigma_{l}^{z} \sigma_{l_{+}}^{z}\right\rangle$ of the integrable model given by (1) have recently been obtained using the quantum inverse scattering method (QISM) by Bogoliubov et al (1986). For $|\Delta| \leqslant 1$ these show a different behaviour for positive and negative $\Delta$. Over the range $0<\Delta<1$ the asymptotic form alternates in sign (antiferromagnetic behaviour) but decays algebraically as $(-1)^{n} n^{\theta}$ with a power $\theta$ in the range $-2<\theta<-1$. For $\Delta$ in the range $-1<\Delta<0$ the asymptotic form does not alternate in sign (ferromagnetic behaviour) but decays algebraically as $n^{-2}$. The significance of this subtle change at $\Delta=0$ is not very clear at present but it is possible that a model state rather different from the antiferromagnetic Néel state might be more appropriate for the range $-1<\Delta<0$.

Finally in this section we relate the LSUBn results given here to earlier results we obtained in I and II using the SUB2 and SUB2 $+g_{4}$ approximations. In the SUB2 approximation scheme all coefficients of terms involving two spin flips are retained and all other coefficients set equal to 0 . The suB2 $+g_{4}$ scheme is the same except 
that one additional coefficient, $g_{4}$, is also retained. In these earlier approximations we found strong evidence for a phase transition at $\Delta=\Delta_{c}$ where $\Delta_{c}$ had the value 0.373 for the former and 0.412 for the latter. After considering the behaviour of the sublattice magnetization, the correlation functions, and the excited-state energies, as well as the ground-state energy, we argued that this phase transition corresponds to the $\Delta=1$ phase transition at which the model becomes critical, even though the actual value of $\Delta_{c}$ is not very close to the exact value of 1 . On the other hand, our LSUB $n$ results appear to give accurate numerical values for $E_{\mathrm{g}} / N$ for much lower values of $\Delta$.

The essential difference between the LSUBn results and the earlier ones is that the LSUB $n$ approximation is designed to handle local contributions to the correlation of the wave function in some detail. The $\mathrm{CCM}$ then automatically constructs longer-range contributions in a systematic but approximate way from these shortrange contributions. Conversely, in the SUB2 and SUB2 $+g_{4}$ approximations the most important long-range contributions are explicitly incorporated but these approximations only include the simplest short-range contributions. Our results suggest that numerically accurate results require detailed consideration of the short-range terms but that, as expected, a true phase change requires the presence of long-range terms explicitly. It is interesting to observe that the $\mathrm{CCM}$ is capable of handling these two very different requirements by means of different approximation schemes. Naturally, an ideal scheme would be one that incorporates both aspects simultaneously and this does at least indicate the direction in which further progress should be sought.

\section{Large- $\Delta$ limit}

In this section we show that the LSUBn approximation includes exactly all contributions to the ground-state energy up to order $\Delta^{-n+1}$. Firstly we note that it is completely straightforward to show directly from $(11 a)-(11 c)$ that the three configuration coefficients retained at the LSUB4 level of approximation have the following asymptotic behaviour in the large- $\Delta$ (Ising) limit:

$$
\begin{aligned}
& b_{1} \underset{\Delta \rightarrow \infty}{\longrightarrow} \frac{1}{2} \Delta^{-1}-\frac{1}{8} \Delta^{-3}+O\left(\Delta^{-5}\right) \quad b_{3} \underset{\Delta \rightarrow \infty}{\longrightarrow} \frac{1}{8} \Delta^{-3}+O\left(\Delta^{-5}\right) \\
& g_{4} \underset{\Delta \rightarrow \infty}{\longrightarrow} \frac{1}{4} \Delta^{-2}+O\left(\Delta^{-4}\right) .
\end{aligned}
$$

The ground-state energy in this approximation is thus given from (8) as

$$
E_{\mathrm{g}} / N \underset{\Delta \rightarrow \infty}{\longrightarrow}-\frac{1}{4} \Delta-\frac{1}{4} \Delta^{-1}+\frac{1}{16} \Delta^{-3}+\mathrm{O}\left(\Delta^{-5}\right) \quad \text { LSUB4. }
$$

To the accuracy quoted, this LSUB4 result agrees with the exact result,

$$
E_{\mathrm{g}} / N \underset{\Delta \rightarrow \infty}{\longrightarrow}-\frac{1}{4} \Delta-\frac{1}{4} \Delta^{-1}+\frac{1}{16} \Delta^{-3}-\frac{1}{256} \Delta^{-7}-\frac{1}{512} \Delta^{-9}+\ldots
$$

obtained by Walker (1959) from the exact solution of Orbach (1958).

Naturally, the same level of accuracy quoted in (14) also arises from a fourth-order perturbation theory calculation taking the Ising limit as the unperturbed Hamiltonian, i.e. by writing (2) as

$$
\mathcal{H}=\Delta\left(\mathcal{H}_{0}+\Delta^{-1} \mathcal{H}_{1}\right)
$$


with $\mathcal{H}_{0}$ the Ising part and $\mathcal{H}_{1}$ the $X Y$ part. We note that the form of the Hamiltonian clearly indicates that only even powers of $\mathcal{H}_{1}$ contribute to the ground-state energy perturbation series.

It is particularly interesting to note that within the LSUB4 approximation only the two configurations with coefficients $b_{1}$ and $g_{4}$ actually contribute to the $\mathrm{O}\left(\Delta^{-3}\right)$ term in the large- $\Delta$ ground-state energy expansion. That is, the configuration with coefficient $b_{3}$ only contributes to the ground-state energy at the $O\left(\Delta^{-5}\right)$ level, for which the LSUB4 approximation is not exact. We note that this is so despite the fact that $b_{3}$ itself has a leading-order behaviour of $\mathrm{O}\left(\Delta^{-3}\right)$. However, it is not difficult to understand why this is the case, since the repeated action of the perturbative $X Y$ part $\mathcal{H}_{1}$ of the Hamiltonian on the model Néel state (which is the ground state of the Ising part $\mathcal{H}_{0}$ ) in our rotated Néel basis is either to create or destroy two adjacent up spins. Thus, to create the configuration with coefficient $b_{3}$ shown in figure 1 requires the action of $\mathcal{H}_{1}$ on the Néel state at least three times. Hence it is clear that the $b_{3}$ configuration can only contribute to the Rayleigh-Schrödinger perturbation series for the ground-state energy at sixth or higher orders.

We also note that there is an infinite sequence of terms that contribute to fourthorder perturbation theory. These arise from intermediate configurations (in the usual perturbation sense) involving two pairs of adjacent spin fips with respect to the Néel state, produced by $\mathcal{H}_{1}$ acting twice, and which are an arbitrary non-zero distance apart (i.e., other than the compact four-spin-flip cluster described by $g_{4}$, which has a different energy eigenvalue with respect to the unperturbed $\mathcal{H}_{0}$ ). In our $C \mathrm{CM}$ description, these are described as two independent nearest-neighbour spin-flip configurations, each described by the coefficient $b_{1}$. We recall (see also Bishop and Kümmel 1987) that the exponential form of the wave function in (3), which lies at the heart of the $\mathrm{CCM}$, is arrived at precisely by such a counting with the correct statistical weight of configurations with multiple numbers of independent sub-configurations.

A similar inspection of the nine configurations that contribute to the LSUB6 approximation shows that those described by the coefficients $b_{5}, f_{0125}$ and $f_{0235}$ can only contribute to the ground-state energy perturbation series at the tenth, eighth and twelfth orders at the lowest, respectively. A detailed calculation based on the nine coupled LSUB6 equations given in appendix 1 confirms that this approximation now gives identical results to the sixth-order perturbation theory, i.e., to confirm the LSUB4 result of (14), together with the additional result that the coefficient of the $\Delta^{-5}$ term is zero, as in (17). The analysis further confirms that only the six configurations with coefficients $b_{1}, b_{3}, g_{4}, f_{0134}, f_{0145}$, and $g_{6}$ actually contribute to the large- $\Delta$ result out to the (now cxact) tcrm of order $\Delta^{-5}$. These configurations are the only ones that can be generated by acting upon the Néel state with $\mathcal{K}_{1}$ three or fewer times.

We have also confirmed explicitly by a rather lengthy calculation that the LSUB8 approximation gives identical results to eight-order perturbation theory, i.e., out to terms of order $\Delta^{-7}$ in (15). Once again, the detailed calculation shows that of the 26 configurations contributing to the LSUB8 approximation shown in figure 1, only the 12 described by coefficients $b_{1}, b_{3}, g_{4}, f_{0134}, f_{0125}, f_{0145}, g_{6}, f_{0156}, f_{012356}, f_{0167}$, $f_{012367}$, and $g_{8}$ actually contribute to this order. The remaining 14 only contribute in higher orders.

Although we have not attempted to construct a rigorous proof, it is clear that similar arguments will be valid at any value of the truncation index $n$. Hence we conclude that the LSUB $n$ approximation for general values of $n$ reproduces exactly the results of $n$ th-order perturbation theory. 
A detailed comparison of the LSUBn results for the ground-state energy with the corresponding results from $n$ th-order perturbation theory (PT $n$ ) which include terms in (15) up to $O\left(\Delta^{-n+1}\right)$, is shown in figure 3 for even values of $n \leqslant 8$. It is clear that for a given value of $n$ the LSUB $n$ results are accurate to considerably lower values of $\Delta$ than the corresponding PT $n$ results. In particular, the range of validity of the LSUB $n$ results certainly extends well below $\Delta=1$ which is a natural boundary for the PT $n$ results, and even to below $\Delta=0$ which is the lowest possible boundary for large- $\Delta$ perturbation theory.

The LSUBn approximation thus represents a natural extension of the PTn approximation. It comprises, in effect, a well defined analytic continuation or resummation of the PTn results, within the context of a systematic hierarchy of approximations. In this sense it may be contrasted with alternative rather ad hoc approaches for extending the range of validity or the accuracy of similar PTn sequences such as, for example, Padé approximants, or similar techniques.

Finally, we may also compare this view of the LSUB $n$ hierarchy of approximations as an extension or generalization of perturbation theory with an analogous view of it (Bishop et al 1992) as a rather natural sequence of generalizations of antiferromagnetic spin-wave theory (Anderson 1952, Oguchi 1960). Whereas in the present case the natural perturbative parameter is $\Delta^{-1}$, in the case of spin-wave theory it is $(2 s)^{-1}$, where $s$ is the spin quantum number.

\section{Conclusions}

The LSUB $n$ sequence of approximations is clearly a practical method of calculating the ground-state energy of quantum spin systems. We have been able to formulate the algebraic generation as well as the numerical solution of the coupled non-linear equations on a computer. This process could undoubtedly be refined and also extended to much more powerful machines.

Our method is valid over a considerably wider range of parameters than perturbation theory and although it does not converge quite as rapidly as perturbation theory for $\Delta>1$, this appears to be due to the rather special form of the perturbation series. Its other advantage over perturbation theory is that it can potentially be used for calculating other quantities such as correlation functions, sublattice magnetization and excited-state energies.

In the light of these encouraging results we intend to apply the method to nonintegrable systems in both $\mathrm{ID}$ and $2 \mathrm{D}$. The latter is, of course, of particular interest in connection with high- $T_{c}$ superconductivity.

\section{Acknowledgment}

One of us (RFB) gratefully acknowledges the support of the Science and Engineering Research Council (UK).

\section{Appendix 1. The nine coupled non-linear equations for isub6}

Using a similar format to that given in section 2 for LSUB4, the nine coupled equations for LSUB6 are: 
(i) $b_{1}$ equation

$$
-1+2 \Delta b_{1}-2 g_{4}-2 f_{0145}-2 f_{0134}+3 b_{1}^{2}-2 b_{1} b_{3}-2 b_{3}^{2}-2 b_{3} b_{5}-2 b_{5}^{2}=0
$$

(ii) $b_{3}$ equation

$$
4 \Delta b_{3}-g_{4}-2 f_{0125}-b_{1}^{2}+4 b_{1} b_{3}-2 b_{1} b_{5}-2 b_{3} b_{5}=0
$$

(iii) $b_{5}$ equation

$$
4 \Delta b_{5}-2 f_{0125}-f_{0235}-2 b_{1} b_{3}-b_{3}^{2}+4 b_{1} b_{5}=0
$$

(iv) $g_{4}$ equation

$$
\begin{gathered}
\Delta g_{4}-\Delta b_{1}\left(b_{1}+2 b_{3}\right)-g_{6}+g_{4}\left(4 b_{1}+b_{3}-b_{5}\right)-b_{1}\left(f_{0125}-f_{0134}\right)-b_{3}\left(f_{0125}+f_{0235}\right. \\
\left.+f_{0134}\right)-b_{5}\left(f_{0125}+f_{0235}\right)+2 b_{1} b_{3}^{2}=0
\end{gathered}
$$

(v) $f_{0125}$ equation

$$
\begin{aligned}
4 \Delta f_{0125}-2 \Delta & b_{1}\left(b_{3}+b_{5}\right)-g_{6}-g_{4}\left(b_{1}-b_{3}-b_{5}\right)+b_{1}\left(8 f_{0125}+f_{0235}-f_{0145}\right) \\
& -b_{3}\left(f_{0235}-f_{0145}+f_{0134}\right)+b_{5}\left(f_{0145}+f_{0134}\right)+2 b_{1} b_{3}^{2}+2 b_{3} b_{5}^{2}+2 b_{3}^{2} b_{5} \\
& +2 b_{1} b_{3} b_{5}=0
\end{aligned}
$$

(vi) $f_{0235}$ equation

$$
\begin{gathered}
3 \Delta f_{0235}-\Delta b_{3}^{2}+g_{4}\left(b_{3}+b_{5}\right)+b_{1}\left(f_{0125}+4 f_{0235}\right)+b_{3} f_{0125}+b_{5} f_{0134}+2 b_{1}^{2} b_{3}+2 b_{3}^{2} b_{5} \\
+2 b_{1} b_{3} b_{5}=0
\end{gathered}
$$

(vii) $f_{0145}$ equation

$$
\begin{gathered}
4 \Delta f_{0145}-4 \Delta b_{3} b_{5}-g_{6}-2 b_{1}\left(f_{0125}-4 f_{0145}-f_{0134}\right)+2 b_{3}\left(f_{0125}-f_{0134}\right) \\
+2 b_{5} f_{0125}+4 b_{1} b_{3}^{2}+4 b_{1} b_{5}^{2}=0
\end{gathered}
$$

(viii) $f_{0134}$ equation

$$
\begin{gathered}
2 \Delta f_{0134}-2 \Delta b_{3}^{2}+g_{4}\left(b_{1}+b_{3}\right)+b_{1}\left(f_{0145}+4 f_{0134}\right)+b_{3}\left(f_{0125}-f_{0145}\right)-b_{5}\left(f_{0125}\right. \\
\left.+f_{0235}\right)+2 b_{1}^{2} b_{3}+2 b_{1} b_{3} b_{5}=0
\end{gathered}
$$

(ix) $g_{6}$ equation

$$
\begin{aligned}
\Delta g_{6}-\Delta\left(2 b_{1} g_{4}\right. & \left.+2 b_{5} g_{4}+4 b_{1} f_{0125}+2 b_{1} f_{0145}+4 b_{3} f_{0235}+6 b_{3} f_{0134}\right)+6 b_{1} g_{6}+b_{5} g_{6} \\
& +g_{4}^{2}+g_{4}\left(3 f_{0125}+f_{0235}+2 f_{0145}+2 f_{0134}\right)+f_{0125}\left(f_{0235}+f_{0145}+2 f_{0134}\right) \\
& +f_{0145} f_{0134}-6 b_{1}^{2} b_{3}^{2}-4 b_{1} b_{3}^{2} b_{5}+2 f_{0125}\left(b_{1} b_{3}+b_{1} b_{5}+b_{3} b_{5}\right) \\
& +2 f_{0235}\left(b_{1} b_{5}+b_{3} b_{5}\right)+2 b_{3}^{2} f_{0145}+2 b_{3} b_{5} f_{0134}+2 b_{5}^{2} g_{4}=0 .
\end{aligned}
$$




\section{Appendix 2. Outline of the computational method}

In order to generate the coupled non-linear equations by computer it is useful to divide the method into simpler steps as follows.

In the $O C M$ we need to calculated $\tilde{\mathcal{H}}$ for the Hamiltonian of (2) where $\tilde{A}$ 目 $\mathrm{e}^{-s} A \mathrm{e}^{s}$ for any operator $A$. Since $\mathrm{e}^{-s} A B \mathrm{e}^{S}=\mathrm{e}^{-s} A \mathrm{e}^{s} \mathrm{e}^{-s} B \mathrm{e}^{s}$, each term in (2) will be the product of two of the operators $\tilde{\sigma}_{l}^{+}, \tilde{\sigma}_{l}^{z}$, and $\tilde{\sigma}_{l}^{-}$. In calculating these we use the familiar nested commutator expansion:

$$
\mathrm{e}^{-S} A \mathrm{e}^{S}=A+[A, S]+\frac{1}{2}[[A, S], S]+\ldots
$$

Since $S$ contains only $\sigma^{+}$operators this sequence will terminate after one, two or three terms for $\tilde{\sigma}_{l}^{+}, \tilde{\sigma}_{l}^{z}$, and $\tilde{\sigma}_{l}^{-}$respectively. Writing $\rho_{l}^{z}=\left[\sigma_{l}^{z}, S\right], \rho_{l}^{-}=\left[\sigma_{l}^{-}, S\right]$, and $\tau_{l}^{-}=\frac{1}{2}\left[\rho_{l}^{-}, S\right]$, then $\tilde{\sigma}_{l}^{+}=\sigma_{l}^{+}, \tilde{\sigma}_{l}^{z}=\sigma_{l}^{z}+\rho_{l}^{z}$ and $\tilde{\sigma}_{l}^{-}=\sigma_{l}^{-}+\rho_{l}^{-}+\tau_{l}^{-}$.

Now using $\sigma_{l}^{-}|\Phi\rangle=0, \sigma_{l}^{z}|\Phi\rangle=-|\Phi\rangle, \sigma_{l}^{z} \sigma_{l}^{+}|\Phi\rangle=\sigma_{l}^{+}|\Phi\rangle$, and (2), we obtain

$$
\begin{aligned}
& \tilde{\mathcal{H}}|\Phi\rangle=-\frac{1}{2} \sum_{l} \sigma_{l}^{+} \sigma_{l+1}^{+}|\Phi\rangle-(\Delta / 4) \sum_{l}\left(\sigma_{l}^{z}+\rho_{l}^{z}\right)\left(\sigma_{l+1}^{z}+\rho_{l+1}^{z}\right)|\Phi\rangle \\
&-\frac{1}{2} \sum_{l}\left(\sigma_{l}^{-}+\rho_{l}^{-}+\tau_{l}^{-}\right)\left(\sigma_{l+1}^{-}+\rho_{l+1}^{-}+\tau_{l+1}^{-}\right)|\Phi\rangle \\
&=-\frac{1}{2} \sum_{l} \sigma_{l}^{+} \sigma_{l+1}^{+}|\Phi\rangle-(\Delta / 4) \sum_{l}\left(1-\rho_{l}^{z}+\sigma_{l}^{z} \rho_{l+1}^{z}+\rho_{l}^{z} \rho_{l+1}^{z}\right)|\Phi\rangle \\
&-\frac{1}{2} \sum_{l}\left(\sigma_{l}^{-} \rho_{l+1}^{-}+\sigma_{l}^{-} \tau_{l+1}^{-}+\rho_{l}^{-} \rho_{l+1}^{-}+\rho_{l}^{-} \tau_{l+1}^{-}+\tau_{l}^{-} \rho_{l+1}^{-}+\tau_{l}^{-} \tau_{l+1}^{-}\right)|\Phi\rangle .
\end{aligned}
$$

The $l=0$ part of each term is then evaluated explicitly. Finally, multiplying on the left by $\langle\Phi| C_{I}$ for the different choices of $C_{I}$ yields the terms in the coupled equations.

\section{References}

Anderson P W 1952 Phys. Rev: 86 694-701

Bethe H A 1931 Z Phys. 71 205-26

Bishop R F 1991 Theur. Chim. Acta 80 95-148

Bishop R F and Kümmel $H 1987$ Phys. Today 40(3) 52-60

Bishop R F, Parkinson J B and Yang Xian 1991a Phys. Rev: B 43 13782-5

— 1991b Phys. Rev: B $449425-43$

1992 Phys. Rev. B submitted

Bogoliubov N M, lzergin A G and Korepin V E 1986 NucL Phys. B 275 687-705

Oguchi T 1960 Phys. Rev. 117 117-23

Orbach R 1958 Phys. Rev. 112 309-16

Roger M and Hetherington J H 1990 Phys. Rev. B 41 200-19

Walker L R 1959 Phys, Rev: 116 1089-90

Yang C N and Yang C P 1966a Phys. Rev. 150 321-7

- 1966b Phys. Rev 150 327-39 